\title{
INVESTIGACIONES
}

\section{El rol de la escuela en formación democrática: diseño de un cuestionario}

\author{
The school's role in democratic education: \\ a questionnaire design
}

\section{Ingrid Sala-Bars ${ }^{a}$, Montserrat Alguacil De Nicolás ${ }^{a}$, Laura Garcia-Raga ${ }^{b}$, Maria-Carme Boqué Torremorell ${ }^{a}$}

\author{
${ }^{a}$ Facultat de Psicologia, Ciències de l'Educació i de l'Esport Blanquerna, Universitat Ramon Llull. \\ ingridsb@blanquerna.url.edu, montserratan@blanquerna.url.edu, mariacarmebt@blanquerna.url.edu \\ ${ }^{b}$ Facultad Filosofía y Ciencias de la Educación. Universitat de València. \\ laura.garcia@uv.es
}

\section{RESUMEN}

El papel de los centros educativos en la formación democrática es fundamental para construir sociedades más equitativas e inclusivas. La literatura destaca la falta de instrumentos de evaluación de estrategias de apoyo en este ámbito con propiedades psicométricas adecuadas. El objetivo del trabajo fue construir el cuestionario "Convivencia y participación democrática en los centros educativos" a partir de la validación por jueces y análisis de fiabilidad mediante un estudio piloto. Respecto a los jueces, participaron 16 expertos en la temática y aspectos metodológicos y en cuanto al pilotaje, se contó con 136 directores de escuela. Se analizó la validez de contenido y fiabilidad del instrumento y los resultados indican una validez de contenido adecuada, con un nivel de correlación entre .6 y .7 en la mayoría de los ítems y una fiabilidad aceptable, con una alfa de Cronbach de .876 para la dimensión de Convivencia y un valor de alfa de Cronbach .886 para la dimensión de Participación. A partir de las valoraciones de los jueces y la prueba piloto, se puso de manifiesto la necesidad de revisar el comportamiento de algunos ítems en función de las correlaciones de cada uno con su subescala.

Palabras claves: Participación, convivencia, centros educativos.

\begin{abstract}
The role of schools in democratic education is essential to build more equitable and inclusive societies. The literature highlights the lack of instruments for evaluating support strategies in this field with adequate psychometric properties. The aim of this work was to create the questionnaire "Coexistence and democratic participation in schools" based on validation by expert judges and reliability analysis through a pilot study. Sixteen experts in the subject and methodological aspects participated as judges, and 136 school principals participated in the pilot study. A content validity analysis and a reliability study of the instrument were carried out. The results indicate an adequate content validity and an acceptable reliability, with a correlation level between .6 and .7 for most of the items and acceptable reliability, with a Cronbach's alpha value of .876 for the Coexistence dimension, and a Cronbach's alpha value of .886 for the Participation dimension. From the judges' evaluations and the pilot test, it became clear that the behavior of some items needed to be revised based on the correlations of each item with its subscale.
\end{abstract}

Key words: Participation, coexistence, schools. 


\section{INTRODUCCIÓN}

Entender que las niñas y los niños son ciudadanos y por ello actores sociales implica que la escuela ha de tener, entre sus finalidades, la de favorecer una participación que posibilite el reconocimiento, la exigencia y la defensa de sus derechos (Saviani, 2018). Por tanto, para que la participación infantil sea efectiva se debe promocionar un rol activo de los menores de 18 años en un clima de respeto y confianza, lo que implica que hablar de participación es hablar de convivencia. Según Santos (2003) participar es aprender a convivir.

Estamos, sin duda, ante un concepto poliédrico que reclama un abordaje multidisciplinar. En nuestro caso, hemos diseñado un cuestionario destinado a equipos directivos de las escuelas, como promotores del Plan de Convivencia, sobre el impulso de estas dos dimensiones: convivencia y participación. Así pues, el propósito de este trabajo es presentar los resultados de la prueba piloto para la validación psicométrica de un nuevo instrumento que identifica el rol de los centros docentes en relación con la convivencia y la participación democráticas de la infancia y la adolescencia en vistas a la inclusión e impulso de la voz de los niños y niñas dentro de la escuela.

\section{MARCO TEÓRICO}

Las competencias para la convivencia y participación democráticas no son un equipamiento natural que todos los seres humanos reciben al nacer, sino que deben aprenderse y desarrollarse. Con la publicación de Democracy and Education, el filósofo y pedagogo progresista norteamericano John Dewey (1916) propuso una educación dirigida a desarrollar la individualidad, el valor del presente, la concepción de un mundo en cambio constante, la adquisición de habilidades como medio y no como fin, la responsabilidad y el espíritu crítico y asentó, asimismo, las bases de una verdadera educación "en" democracia capaz de beneficiar bidireccional e indisociablemente la dialéctica entre el individuo y la sociedad, puesto que no hay democracia sin educación y no es posible hablar de educación sin democracia. Además, como más tarde afirmaría Freinet (1996) en la invariante pedagógica núm. 27: "la democracia del mañana se prepara con la democracia en la escuela. Un régimen autoritario en la escuela no sería capaz de formar ciudadanos demócratas". Queda claro, pues, desde sus inicios, que la convivencia y la participación democráticas se aprenden aunando formación y práctica, escuela y contexto social.

Para comprender el presente, es necesario señalar que el pensamiento de Locke, Rousseau, Tolstoi, Korczak, Freinet, Ferrer-Guàrdia, Neill, Makarenko, Freire, el mismo Dewey y demás predecesores de la introducción de la democracia en los centros educativos fue adoptado por la escuela moderna y activa y por los movimientos de renovación pedagógica que le han dado continuidad (Martínez-Bonafé, 2016). Por otra parte, en el ámbito de la Unión Europea se ha venido manifestando de manera explícita y sostenida el interés por educar en convivencia y participación democráticas a través de varias iniciativas, entre las más actuales merece la pena señalar: las "Competencias clave para un aprendizaje a lo largo de la vida" (Comisión Europea, 2004), que incluyen tanto las competencias interpersonales, interculturales y sociales como las competencias cívicas; la declaración del año 2005 como "Año Europeo de la Ciudadanía a través de la Educación" (Eurydice, 2005), cuyos objetivos fueron impulsar una política educativa para la ciudadanía 
democrática y la inclusión social, promover el papel y las competencias docentes en la formación para la ciudanía y fomentar las formas de gobierno democráticas en los centros; la "Carta del Consejo de Europa sobre la educación para la ciudanía democrática y la educación en derechos humanos" (Consejo de Europa, 2010), que incide una vez más en el papel esencial de la educación en la promoción de la democracia; la "Recomendación del Consejo de Europa sobre la participación de los niños y jóvenes de menos de 18 años de edad" (Consejo de Europa, 2012), donde se exhorta a los países miembros a proporcionar oportunidades a todos los niños y niñas para participar en lo que les afecta; la "Declaración sobre la promoción de la ciudadanía y de los valores comunes de libertad, tolerancia y no discriminación mediante la educación" (Consejo de Europa, 2015) y la "Recomendación relativa a la promoción de los valores comunes, la educación inclusiva y la dimensión europea de la enseñanza" (Consejo de Europa, 2018), ambas en la línea de dar respuesta a los ataques extremistas sufridos en distintos países europeos a través de medidas preventivas y educativas; o el programa "Horizonte 2020" (Unión Europea, 2014), con acciones concretas para la construcción de sociedades inclusivas, participativas y abiertas aprovechando el potencial innovador, creativo y productivo de todas las generaciones.

Estas políticas se hallan en total consonancia con otras normativas y programas de alcance mundial como la "Convención sobre los derechos del niño", promulgada por la Asamblea General de la Organización de las Naciones Unidas (ONU, 1989), que respalda el derecho de la infancia de participar con voz propia a través de 54 artículos sustentados en cuatro principios: la no discriminación, el interés superior del niño, el derecho a la vida, a la supervivencia y al desarrollo y el respeto de sus opiniones. Autores como Tonucci (1996, 2003, 2009) han manifestado reiteradamente su compromiso con la defensa de los derechos de la infancia y, muy específicamente, con el de ser parte y tomar parte en la escuela y en el entorno como nuevo agente social y socio en democracia, insistiendo en que "esta no es una experiencia educativa sino política, se lleva adelante por la ciudad, no por los niños" (Tonucci et al., 2015, p. 69). También Moss (2013, 2014) y Cameron y Moss (2020) reclaman una concepción comprensiva de la infancia que incluya a los más pequeños y deje de considerarles simplemente como "futuro capital humano". Su reflexión es interesante porque repiensa la educación a través de la autonomía, la diversidad, la participación, la confianza, etc. Esta forma de entender la participación infantil, de acuerdo con Belavi y Murillo (2016) y Murillo y Hernández-Castilla (2014), está íntimamente relacionada con el concepto de justicia social y requiere una educación equitativa, crítica y democrática abocada a la construcción de un mundo más justo.

Más recientemente, la "Agenda 2030 sobre el Desarrollo Sostenible" (ONU, 2015), traza un plan de trabajo alrededor de 17 objetivos para lograr un mundo más habitable $\mathrm{y}$, concretamente, la meta 4.7 puntualiza que se ha de asegurar que todo el alumnado adquiera los conocimientos teóricos y prácticos necesarios para promover el desarrollo de los derechos humanos, la igualdad de género, la promoción de una cultura de paz, la ciudadanía mundial y la contribución a un desarrollo sostenible. A su vez, la Organización para la Cooperación y el Desarrollo Económico lanza el "Learning Compass 2030" (OECD, 2020), la brújula del aprendizaje, como marco para la formación integral que establece las nuevas competencias para desarrollarse como persona poniendo, ahora, en valor las competencias transformacionales —que incluyen la creación de nuevos valores, la reconciliación de tensiones y dilemas y la asunción de responsabilidad-y las competencias globales necesarias para ejercer una ciudadanía responsable y ser agentes del cambio. 
Las anteriores directrices han tenido, como cabía esperar, repercusión en la legislación educativa de los distintos países, también en España y en las respectivas comunidades autónomas que han desplegado normativas que regulan la convivencia de una manera cada vez más proactiva, tal y como analizan varios estudios (García-Raga y López, 2014; Gómez, 2011). También desde la perspectiva de la escuela inclusiva se identifican la convivencia y la participación como factores clave, ya que sin un clima relacional positivo y sin la capacidad de intervenir activamente no se podría considerar verdadera inclusión (Peñalva y Vega, 2019).

Por lo que se refiere específicamente a la convivencia democrática, la obligatoriedad, por parte de las administraciones educativas, de que las escuelas implementen un Plan de Convivencia incrementa la necesidad de los centros docentes de contar con herramientas y recursos que les ayuden a la hora de dar respuesta a sus necesidades, entendiendo por convivencia en la escuela las relaciones que se dan en los centros educativos a nivel individual y a nivel institucional (Ochoa y Diez-Martínez, 2012; Ochoa y Salinas, 2016). Por su parte, Carrillo y Feu (2015) y Reddy y Ratna (2002), articulan investigaciones en torno a que los menores de 18 años no son seres pasivos respecto a temas relacionados con su propia vida, sino que tienen interés y se implican en el debate sobre la mejora de las condiciones de la propia infancia. También, diferentes iniciativas relacionadas no solo con la participación escolar, sino también en otros ámbitos, desarrollan objetivos para empoderar a los menores de 18 años, entre las que destacaríamos "Save the Children" (2016), que incorpora la gobernanza infantil entre sus ejes prioritarios, o los estudios que analizan el papel de la participación infantil en el ámbito del bienestar y la protección (Dillon et al., 2015; Kosher et al., 2016).

Como hemos comentado, participación y convivencia son dos caras de la misma moneda en el marco de una sociedad democrática y se espera de las escuelas que estimulen el deseo del alumnado de contribuir activa y cívicamente a la cohesión social (Wanders et al., 2020). Liwski (2006), a su vez, considera que la participación y la convivencia deberían guiarse por tres propósitos fundamentales: desarrollo de aprendizajes que permitan a la infancia asumir su condición de ciudadanos y ciudadanas de pleno derecho, solidarios y responsables con su entorno social; promoción del sentido de pertenencia y de un estilo de vida basado en la confianza y la participación; y creación de una cultura democrática que elimine la exclusión y la discriminación en todas sus formas. Mayoritariamente, estos referentes apuntan en la línea del llamado "enfoque orientado en el sujeto" de Liebel (2007) que concibe al niño como una persona competente y con derecho de acción.

Sin lugar a duda, un ámbito privilegiado para promover la convivencia y la participación es la escuela, ya que supone un espacio socializador secundario (después de la familia) para todos los niños y las niñas. Así, Gil-Jaurena et al. (2013) y Martín Bris y Gairín (2007), entre otros, defienden una visión comunitaria de los centros docentes como impulsores de un proyecto cultural compartido con los servicios y entidades del entorno, el cual permite desarrollar acciones conjuntas y aprovechar mejor los recursos y supone un foco aglutinador para la implicación social. Además, Bolívar (2016) cree que la participación infantil supone un elemento constitutivo de la escuela democrática que permite a todo el alumnado ejercer su ciudadanía activa, entendiendo que la educación que no tiene lugar en un contexto democrático deja de ser educación para convertirse en instrucción y, en el peor de los casos, adoctrinamiento. Para Boque et al. (2017) los beneficios del desarrollo de la convivencia y de la participación infantil se pueden 
clasificar, como mínimo, en tres dimensiones: jurídica, sociopolítica y pedagógica. En cuanto a estrategias democratizadoras, Moliner et al. (2016) señalan la creación de una cultura colaborativa, la lucha contra la exclusión y el favorecimiento de la diversidad como fuente de mejora, la redefinición de un conjunto de valores democráticos, la facilitación de la toma de decisiones de la comunidad, la investigación-acción como estrategia de mejora, el empoderamiento y la autodeterminación, el desarrollo de procesos formativos sobre participación democrática y desarrollo de proyectos compartidos entre el centro educativo y su territorio.

Justamente, la no discriminación en el marco de la convivencia basada en la inclusión es la base de estudios como, por ejemplo, los de Alderson (2010), centrados en el derecho a participar de las niñas y niños más pequeños, o los de Ray (2010), quien estudia la participación de los menores en situación de pobreza. La necesidad de asegurar la participación de menores de 18 años con alguna discapacidad es abordada, entre otros, por Fielding (2011), Franklin y Sloper (2004) y Griffiths et al. (1999). Dadich (2010) reivindica la inclusión en los mecanismos de participación de niños y niñas con problemas de salud mental. También hay referentes que analizan la dimensión participativa de los migrantes no acompañados (Save the Children, 2016; Thomas, 2009), e incluso autores como Feinstein et al. (2010) abordan la dimensión participativa de los pequeños en situación de conflicto armado. En todos estos estudios y otros similares se acentúa la capacidad de la infancia de organizarse y luchar por la propia autonomía, convirtiéndose en verdaderos actores políticos, implicados y comprometidos con la realidad que los rodea.

A partir de estos postulados, entendemos la escuela como espacio de convivencia y dinamizadora de políticas de participación de gran alcance, tanto en la vertiente formativa como vivencial, desarrollando una preparación cívica que incorpora un concepto de inclusión enmarcado en el paradigma del diseño universal (Cook y Rao, 2018; Echeita y Ainscow, 2011; Rao et al., 2017; Sala et al., 2014). Desde este marco referencial, el diseño de un cuestionario para valorar la convivencia democrática y la participación infantil en la escuela y su proyección pública, a partir de la visión de los equipos directivos, quiere contribuir al paso de una visión asistencial de la infancia, aún en parte vigente, a una concepción basada en los derechos y la dignidad de todas las personas.

De entre los diferentes instrumentos diseñados en nuestro entorno como por ejemplo los de Martín Babarro et al. (2010), promovidos por el Ministerio de Educación y Ciencia, con indicadores globales sobre convivencia escolar, los de Del Rey et al. (2017) referidos a la evaluación del clima de convivencia dirigidos al alumnado, al profesorado y a las familias, el CUVE-R (Álvarez-García et al., 2011) sobre violencia escolar, y el de Pérez Galván y Ochoa (2018), que valora su posición sobre temas controvertidos como la libertad de expresión o la participación infantil, ninguno aúna factores de convivencia e impulso de la participación en el contexto escolar en el marco de una escuela democrática inclusiva, a partir de la visión de los directores, entendidos como líderes del proyecto educativo del centro y ésta es precisamente la finalidad del instrumento que proponemos. 


\section{MÉTODO}

La muestra del estudio está compuesta por de 136 directores de escuelas de la comunidad autónoma de Catalunya (España). Concretamente, un $83.2 \%$ responden a escuelas de titularidad pública (escuelas que reciben exclusivamente fondos públicos) y un $16.8 \%$ de titularidad concertada (escuelas privadas que tiene enseñanzas que reciben fondos públicos). En relación con la cualificación de estos centros, el $73.7 \%$ estaban cualificados como centros ordinarios (escuelas que dan respuesta educativa a las necesidades de toda la diversidad de alumnado), el $18.2 \%$ como centros de complejidad (escuelas que concentran una elevada proporción de alumnado socialmente desfavorecido) y el $8.1 \%$ tenían otros tipos de cualificación. Cabe remarcar que los Centros de Educación Especial (escuelas exclusivas para niños y niñas con discapacidad), dadas sus características particulares, no fueron incluidos en la muestra invitada. En relación con el perfil de los directores, del total de participantes el $83.2 \%$ eran mujeres, el $28.5 \%$ eran hombres y el $0.7 \%$ prefiere no contestar con relación al género. En cuanto a los años que llevaban trabajando en el centro, el $62.8 \%$ declaró más de diez años, el $24.8 \%$ entre cinco y diez años y el $12.4 \%$ menos de cinco años. Referente a su experiencia en dirección de centros, el $40.9 \%$ tenía menos de cinco años de experiencia en dirección, el $29.9 \%$ entre cinco y diez años de experiencia y el $29.2 \%$ más de diez años. En cuanto a la formación de los participantes en temas de convivencia, el $93.4 \%$ había recibido algún tipo de formación sobre convivencia mientras que el $6.6 \%$ no había recibido ninguna formación sobre el tema. Sobre la formación en temas de participación de la infancia y adolescencia, el 61.3\% había recibido algún tipo de formación y el 38.7\% no había recibido ningún tipo de formación sobre este tema. Acerca de la formación de la gestión de la diversidad en el marco de una escuela para todos y todas, el $88.3 \%$ de los participantes había recibido formación y el $11.7 \%$ no la había recibido.

En este estudio también participaron 16 expertos que llevaron a cabo la valoración del cuestionario. Seis de ellos eran expertos en temas de participación de la infancia y la adolescencia, uno en temas de escuela inclusiva y atención a la diversidad, cinco en metodología e investigación y, finalmente, cuatro eran directores de escuela.

El instrumento utilizado es el cuestionario "Convivencia y participación democrática en los centros educativos". Los ítems se han creado a partir de la revisión exhaustiva de la literatura actual y complementándolos con las aportaciones de los expertos tanto del ámbito profesional como de investigación que se dedican a temas vinculados a la participación de la infancia y adolescencia y a la atención a la diversidad.

El cuestionario está dividido en cuatro grandes apartados y un apartado final de Otras observaciones (tabla 1). Además, el tercer y cuarto apartado están constituidos, cada uno de ellos, por diferentes subapartados. Todo esto con la intención de poder evaluar los objetivos propuestos. 
Tabla 1. Apartados del cuestionario

"Convivencia y participación democrática en los centros educativos"

\begin{tabular}{|c|c|c|}
\hline \multicolumn{2}{|r|}{ Apartados y subapartados } & \multirow{2}{*}{$\frac{\mathrm{N}^{\mathrm{o}} \text { Ítems }}{4}$} \\
\hline 1. & Datos generales del centro & \\
\hline 2. & Datos generales de la dirección del centro & 7 \\
\hline \multirow[t]{6}{*}{3.} & Ámbito de convivencia democrática & 28 \\
\hline & 3.1 Formación: desarrollo de competencias para aprender a vivir juntos & 6 \\
\hline & 3.2 Prácticas: programas y acciones para aprender a vivir juntos & 4 \\
\hline & 3.3 Funcionamiento: gestión de la convivencia en el centro & 6 \\
\hline & $\begin{array}{l}\text { 3.4 Acceso Universal: aplicación de los principios inclusivos en las } \\
\text { relaciones interpersonales }\end{array}$ & 6 \\
\hline & $\begin{array}{l}\text { 3.5 Proyección: compromiso del centro en el ámbito de la convivencia y } \\
\text { detección de necesidades }\end{array}$ & 6 \\
\hline \multirow[t]{6}{*}{4.} & Ámbito de participación democrática & 28 \\
\hline & 4.1 Formación: desarrollo de competencias para una cultura democrática & 5 \\
\hline & 4.2 Prácticas: programas y actuaciones para una cultura democrática & 5 \\
\hline & 4.3 Funcionamiento: gestión de la participación en el centro & 6 \\
\hline & $\begin{array}{l}\text { 4.4 Acceso Universal: aplicación de los principios inclusivos a la } \\
\text { participación democrática }\end{array}$ & 6 \\
\hline & $\begin{array}{l}\text { 4.5 Proyección: compromiso del centro en el ámbito de la participación y } \\
\text { detección de necesidades }\end{array}$ & 6 \\
\hline 5. & Otras observaciones & N/A \\
\hline
\end{tabular}

La primera parte "Datos generales del centro" está compuesta por cuatro ítems a través de los que se evalúan los aspectos vinculados a las características del centro educativo (titularidad, tipología, número de alumnos y número de habitantes del municipio donde está ubicado el centro). Estas preguntas son de respuesta múltiple y todas ellas deben ser contestadas por el director o directora del centro.

La segunda parte "Datos Generales de la Dirección del Centro" recoge la información relacionada con el género, la edad, los años de experiencia docente en el centro, los años de experiencia en la dirección del centro, y la formación en convivencia, en participación y en atención a la diversidad del responsable del centro. Concretamente, son siete ítems de respuesta múltiple que contesta el director o directora del centro.

La tercera parte "Ámbito de convivencia democrática" sirve para conocer el rol de los centros en cuanto a la formación para la convivencia del colectivo infantil y adolescente en vista a la inclusión e impulso de la voz de los niños y niñas dentro de los centros educativos. En total, esta parte consta de 28 ítems formulados en escalas de Likert que están divididos en cinco subcomponentes. El primer subcomponente "Formación: desarrollo de 
competencias para aprender a vivir juntos" incluye seis ítems y tiene por objetivo identificar las acciones formativas de los centros docentes en el desarrollo de las competencias para aprender a vivir juntos. El segundo subcomponente "Prácticas: programas y acciones para aprender a vivir juntos" incluye cuatro ítems y tiene por objetivo identificar las prácticas de los centros docentes en el desarrollo de las competencias para aprender a vivir juntos. El tercer subcomponente "Funcionamiento: gestión de la convivencia en el centro" incluye seis ítems que tienen por objetivo identificar la organización del centro en el desarrollo de las competencias para aprender a vivir juntos. El cuarto subcomponente "Acceso Universal: aplicación de los principios inclusivos en las relaciones interpersonales" consta de seis ítems y tiene por objetivo identificar las acciones que promueven la accesibilidad en cuestión de convivencia a todos los niños y adolescentes, así como las barreras que la dificultan. El quinto subcomponente "Proyección: compromiso del centro en el ámbito de la convivencia y detección de necesidades" formado por seis ítems y tiene por objetivo detectar la disponibilidad de los centros para promover la convivencia positiva en el centro y colaborar en fórums de convivencia de niños y adolescentes en el entorno.

La cuarta parte del cuestionario "Ámbito de participación democrática" sirve para conocer el rol de los centros en cuanto a la participación democrática del colectivo infantil y adolescente en vista a la inclusión e impulso de la voz de los niños y las niñas dentro de los centros educativos y en la administración pública. En total, esta parte consta de 28 ítems formulados en escalas de Likert que están divididos en cinco subcomponentes. El primer subcomponente "Formación: desarrollo de competencias para una cultura democrática" incluye cinco ítems y tiene por objetivo identificar las acciones formativas de los centros docentes en el desarrollo de las competencias para el ejercicio de la participación democrática. El segundo subcomponente "Prácticas: programas y actuaciones para una cultura democrática" consta de incluye cinco ítems y tiene por objetivo identificar las prácticas de los centros docentes en el desarrollo de las competencias para el ejercicio de la participación democrática. El tercer subcomponente "Funcionamiento: gestión de la participación en el centro" formado por 6 ítems que tienen por objetivo identificar la organización del centro en el ejercicio de la participación democrática por parte del alumnado. El cuarto subcomponente "Acceso Universal: aplicación de los principios inclusivos a la participación democrática" incluye seis ítems y tiene por objetivo identificar las acciones que promueven la accesibilidad a la participación a todos los niños y adolescentes, así como las barreras que la dificultan. El quinto subcomponente "Proyección: compromiso del centro en el ámbito de la participación y detección de necesidades" consta de seis ítems y tiene por objetivo detectar la disponibilidad de los centros en colaborar en fórums de participación de niños y adolescentes dentro de la administración pública.

Finalmente, el instrumento contiene un último apartado de "Otras observaciones" donde los participantes, si quieren, pueden añadir otras cuestiones con relación a la temática tratada que no han quedado recogidas en el instrumento o que le han interpelado.

En relación al procedimiento, inicialmente se creó un banco de ítems a partir de la literatura más relevante en relación con el tema de la convivencia y la participación democrática de la infancia y la adolescencia en el ámbito educativo (Boqué et al., 2014; Carbonell et al., 2018; Dillon et al., 2015; Kosher et al., 2016; Santibáñez et al., 2015; Toro, 2011; Trilla y Novella, 2011;) y los que tratan específicamente el acceso universal e identificación de barreras (Cook y Rao, 2018; Echeita y Ainscow, 2011; Rao et al., 2017; 
Sala et al., 2014). Después de elaborar los 67 ítems, se procedió a analizar su validez de contenido y fiabilidad. En cuanto a la validez de contenido, el análisis se llevó a cabo mediante la técnica de juicio de expertos. En concreto, el instrumento se sometió a la valoración de dieciséis expertos en el ámbito de la convivencia, la participación y la inclusión educativa para que los revisaran. Mediante un correo electrónico, se envió una carta de presentación, indicando el objetivo del instrumento, la importancia de su colaboración y el agradecimiento del equipo investigador. Al mismo tiempo, se entregaron instrucciones para completar la evaluación de cada ítem según la descripción de la dimensión respectiva. Concretamente, se les pedía a que valoraran, a partir de un formulario con escala Likert graduada de 1 (no cumple) a 4 (alto cumplimiento), la suficiencia, la claridad, la coherencia y la relevancia de cada ítem, además de revisar la estructura del cuestionario. También se proporcionó una sección para que el experto realizara los comentarios pertinentes, ya sea sobre la redacción de algún ítem o la dificultad para su comprensión.

Una vez obtenidas las respuestas, nos interesaban dos aspectos. Por un lado, cuáles eran las valoraciones y observaciones que los jueces habían realizado de los ítems considerando los criterios anteriormente señalados y cuál era el grado de acuerdo entre los mismos. En primer lugar, respecto a la valoración de los ítems, cabe indicar que las puntuaciones fueron muy altas y cercanas al 4 (alto cumplimiento) por parte de los 16 jueces. Por otra parte, para calcular el grado de acuerdo entre los jueces, se utilizó el coeficiente de concordancia W de Kendall. Los resultados obtenidos señalan un nivel de correlación situado entre 0.6 y 0.7 en la mayoría de los ítems, lo que indica un buen grado de concordancia en las valoraciones, sin detectarse diferencias significativas entre las respuestas ofrecidas por los jueces. Se decidió, por tanto, no eliminar ningún ítem, si bien se realizan algunas reformulaciones menores en función de las observaciones aportadas para mejorar validez.

Tras analizar las aportaciones del juicio de expertos e introducir las modificaciones pertinentes, se realizó una prueba piloto con el objetivo de analizar la consistencia interna del cuestionario.

Para llevar a cabo esta prueba piloto, en una primera fase, se envió el instrumento a una muestra aleatoria de 500 centros educativos de Cataluña con un recordatorio después de quince días. En una segunda fase, para incrementar el número de respuestas, se hizo un segundo envío a 400 centros educativos de Cataluña que pertenecían a una muestra de reserva. Finalmente, participaron un total de 136 directores y directoras de escuelas e institutos, lo cual representa un $15.1 \%$ del total de los centros donde se envió el cuestionario.

Por último, partir de los resultados obtenidos del pilotaje, se procedió al cálculo de la consistencia interna mediante el coeficiente alfa de Cronbach.

\section{RESULTADOS}

Los datos que aquí se presentan deben solo interpretarse como una aproximación, derivados de la prueba piloto efectuada, sin que puedan usarse como valores asociados al estudio psicométrico que la versión definitiva del cuestionario debe superar.

Se procedió al cálculo del coeficiente de Cronbach de cada componente del instrumento. Los valores de este coeficiente oscilan entre 0 y 1 , considerando como criterio general un coeficiente aceptable cuando su valor es igual o superior a 0.70 (Nunally, 1975). A continuación, se exponen los resultados obtenidos en cada uno de ellos. 


\subsection{COMPONENTE CONVIVENCIA}

Para este componente del cuestionario y dado que se trata de ítems formulados en escalas de Likert, se procedió a un mínimo estudio de fiabilidad desde la Teoría Clásica de los Test, obteniéndose buenos resultados. En concreto, un valor de a de Cronbach de .876, claramente indicativo de una elevada consistencia interna.

\subsubsection{Formación: desarrollo de competencias para aprender a vivir juntos}

La fiabilidad de este subcomponente se calculó también mediante el coeficiente alfa de Cronbach. Para esta parte se obtuvo un valor de .810. Este resultado nos permite afirmar que los ítems miden de forma consistente los elementos que se pretenden estudiar. En la tabla 2 se muestra la necesidad de todos los ítems.

Tabla 2. Análisis de cada ítem con el total del subcomponente "Formación: desarrollo de competencias para aprender a vivir juntos"

\begin{tabular}{|l|c|c|c|c|}
\hline & $\begin{array}{c}\text { Media de escala si } \\
\text { el elemento se ha } \\
\text { suprimido }\end{array}$ & $\begin{array}{c}\text { Varianza de escala } \\
\text { si el elemento se ha } \\
\text { suprimido }\end{array}$ & $\begin{array}{c}\text { Correlación total } \\
\text { de elementos } \\
\text { corregida }\end{array}$ & $\begin{array}{c}\text { Alfa de Cronbach } \\
\text { si el elemento se ha } \\
\text { suprimido }\end{array}$ \\
\hline Ítem 1 & 17.08 & 5.542 & .453 & .805 \\
\hline Ítem 2 & 16.83 & 5.267 & .669 & .761 \\
\hline Ítem 3 & 17.08 & 5.127 & .569 & .780 \\
\hline Ítem 4 & 16.89 & 5.195 & .603 & .772 \\
\hline Ítem 5 & 17.21 & 4.965 & .547 & .788 \\
\hline Ítem 6 & 16.75 & 5.419 & .619 & .772 \\
\hline
\end{tabular}

\subsubsection{Prácticas: programas y acciones para aprender a vivir juntos}

Para este subcomponente se obtuvo un bajo coeficiente de fiabilidad, con un valor alfa de Cronbach de .679. En la tabla 3 se aprecia el análisis de cada ítem. Esto implica que este subcomponente será revisado para la versión definitiva. 
Tabla 3. Análisis de cada ítem con el total del subcomponente

"Prácticas: programas y acciones para aprender a vivir "juntos"

\begin{tabular}{|l|c|c|c|c|}
\hline & $\begin{array}{c}\text { Media de escala si } \\
\text { el elemento se ha } \\
\text { suprimido }\end{array}$ & $\begin{array}{c}\text { Varianza de escala } \\
\text { si el elemento se } \\
\text { ha suprimido }\end{array}$ & $\begin{array}{c}\text { Correlación total } \\
\text { de elementos } \\
\text { corregida }\end{array}$ & $\begin{array}{c}\text { Alfa de Cronbach } \\
\text { si el elemento se } \\
\text { ha suprimido }\end{array}$ \\
\hline Ítem 1 & 10.21 & 5.725 & .509 & .583 \\
\hline Ítem 2 & 10.24 & 5.844 & .451 & .620 \\
\hline Ítem 3 & 10.43 & 5.076 & .460 & .625 \\
\hline Ítem 4 & 10.21 & 6.613 & .456 & .625 \\
\hline
\end{tabular}

\subsubsection{Funcionamiento: gestión de la convivencia en el centro}

Para esta parte se obtuvo un valor de .716. Este resultado nos permite afirmar que los ítems miden de forma consistente los elementos que se pretenden estudiar. En la tabla 4 se muestra el análisis de cada ítem que no resulta especialmente elevado. En este caso, es más que probable que un aumento del tamaño de la muestra permita obtener mejores resultados.

Tabla 4. Análisis de cada ítem con el total del subcomponente

"Funcionamiento: gestión de la convivencia en el centro"

\begin{tabular}{|l|c|c|c|c|}
\hline & $\begin{array}{c}\text { Media de escala si } \\
\text { el elemento se ha } \\
\text { suprimido }\end{array}$ & $\begin{array}{c}\text { Varianza de escala } \\
\text { si el elemento se } \\
\text { ha suprimido }\end{array}$ & $\begin{array}{c}\text { Correlación total } \\
\text { de elementos } \\
\text { corregida }\end{array}$ & $\begin{array}{c}\text { Alfa de Cronbach } \\
\text { si el elemento se } \\
\text { ha suprimido }\end{array}$ \\
\hline Ítem 1 & 14.56 & 6.471 & .432 & .684 \\
\hline Ítem 2 & 14.44 & 6.708 & .425 & .684 \\
\hline Ítem 3 & 13.18 & 6.709 & .521 & .657 \\
\hline Ítem 4 & 13.22 & 6.929 & .533 & .659 \\
\hline Ítem 5 & 13.24 & 6.833 & .452 & .676 \\
\hline Ítem 6 & 13.57 & 6.587 & .376 & .704 \\
\hline
\end{tabular}

4.1.4. Acceso universal: aplicación de los principios inclusivos en las relaciones interpersonales

El coeficiente de fiabilidad indicó una elevada consistencia interna, con un valor alfa de .803. Los valores de discriminabilidad para cada ítem presentados en la tabla 5 indican que ninguno de ellos debería ser eliminado en la versión definitiva del cuestionario. 
Tabla 5. Análisis de cada ítem con el total del subcomponente

"Accesibilidad Universal: aplicación de los principios inclusivos en las relaciones interpersonales"

\begin{tabular}{|l|c|c|c|c|}
\hline & $\begin{array}{c}\text { Media de escala si } \\
\text { el elemento se ha } \\
\text { Suprimido }\end{array}$ & $\begin{array}{c}\text { Varianza de escala } \\
\text { si el elemento se } \\
\text { ha suprimido }\end{array}$ & $\begin{array}{c}\text { Correlación total } \\
\text { de elementos } \\
\text { corregida }\end{array}$ & $\begin{array}{c}\text { Alfa de Cronbach } \\
\text { si el elemento se } \\
\text { ha suprimido }\end{array}$ \\
\hline Ítem 1 & 17.60 & 5.397 & .422 & .810 \\
\hline Ítem 2 & 17.60 & 5.708 & .484 & .788 \\
\hline Ítem 3 & 17.80 & 5.553 & .498 & .786 \\
\hline Ítem 4 & 17.71 & 5.276 & .606 & .762 \\
\hline Ítem 5 & 17.66 & 5.144 & .706 & .741 \\
\hline Ítem 6 & 17.84 & 4.907 & .682 & .742 \\
\hline
\end{tabular}

\subsubsection{Proyección: compromiso del centro en el ámbito de la convivencia y detección de necesidades}

En este subcomponente se obtuvo un valor de .769 . Este resultado nos permite afirmar que los ítems miden de forma consistente los elementos que se pretenden estudiar. En la tabla 6 se muestra que ninguno de ellos debería ser eliminado en la versión definitiva del cuestionario.

Tabla 6. Análisis de cada ítem con el total del subcomponente

"Proyección: compromiso del centro en el ámbito de la convivencia y detección de necesidades"

\begin{tabular}{|l|c|c|c|c|}
\hline & $\begin{array}{c}\text { Media de escala si } \\
\text { el elemento se ha } \\
\text { suprimido }\end{array}$ & $\begin{array}{c}\text { Varianza de escala } \\
\text { si el elemento se } \\
\text { ha suprimido }\end{array}$ & $\begin{array}{c}\text { Correlación total } \\
\text { de elementos } \\
\text { corregida }\end{array}$ & $\begin{array}{c}\text { Alfa de Cronbach } \\
\text { si el elemento se } \\
\text { ha suprimido }\end{array}$ \\
\hline Ítem 1 & 14.12 & 6.875 & .514 & .734 \\
\hline Ítem 2 & 12.03 & 6.636 & .613 & .708 \\
\hline Ítem 3 & 13.85 & 6.887 & .621 & .709 \\
\hline Ítem 4 & 13.72 & 6.855 & .599 & .713 \\
\hline Ítem 5 & 13.71 & 6.784 & .555 & .723 \\
\hline Ítem 6 & 13.33 & 7.867 & .231 & .809 \\
\hline
\end{tabular}




\subsection{COMPONENTE PARTICIPACIÓN}

Para este componente del cuestionario también se estudió de fiabilidad desde la Teoría Clásica de los Test, obteniéndose buenos resultados. En concreto un valor de a de Cronbach de .886 , claramente indicativo de una elevada consistencia interna. Sin embargo, pese a este resultado global, se decidió para una mayor precisión analizar la consistencia interna de cada uno de los subcomponentes o categorías.

\subsubsection{Formación: desarrollo de competencias para una cultura democrática}

La fiabilidad de este subcomponente se calculó también mediante el coeficiente alfa de Cronbach y se obtuvo un valor de .848 . Este resultado nos permite afirmar que los ítems miden de forma consistente los elementos que se pretenden estudiar. En la tabla 7 se muestra la necesidad de todos los ítems.

Tabla 7. Análisis de cada ítem con el total del subcomponente

"Formación: desarrollo de competencias para una cultura democrática"

\begin{tabular}{|l|c|c|c|c|}
\hline & $\begin{array}{c}\text { Media de escala si } \\
\text { el elemento se ha } \\
\text { suprimido }\end{array}$ & $\begin{array}{c}\text { Varianza de escala } \\
\text { si el elemento se } \\
\text { ha suprimido }\end{array}$ & $\begin{array}{c}\text { Correlación total } \\
\text { de elementos } \\
\text { corregida }\end{array}$ & $\begin{array}{c}\text { Alfa de Cronbach } \\
\text { si el elemento se } \\
\text { ha suprimido }\end{array}$ \\
\hline Ítem 1 & 10.70 & 5.145 & .680 & .812 \\
\hline Ítem 2 & 10.54 & 5.125 & .660 & .816 \\
\hline Ítem 3 & 11.05 & 4.790 & .705 & .804 \\
\hline Ítem 4 & 10.53 & 4.873 & .723 & .800 \\
\hline Ítem 5 & 11.71 & 5.006 & .543 & .852 \\
\hline
\end{tabular}

\subsubsection{Prácticas: programas y actuaciones para aprender a una cultura democrática}

Para este subcomponente se obtuvo un valor alfa de Cronbach de .748. En la tabla 8 se aprecia el análisis de cada ítem. Los resultados indican que la mayoría de los ítems miden de forma consistente los elementos que se pretenden estudiar. Los ítems 2 y 3 obtienen valores más bajos, pero un aumento del tamaño de la muestra permitirá obtener mejores resultados. 
Tabla 8. Análisis de cada ítem con el total del subcomponente

"Prácticas: programas y actuaciones para aprender a una cultura democrática"

\begin{tabular}{|l|c|c|c|c|}
\hline & $\begin{array}{c}\text { Media de escala si } \\
\text { el elemento se ha } \\
\text { suprimido }\end{array}$ & $\begin{array}{c}\text { Varianza de escala } \\
\text { si el elemento se } \\
\text { ha suprimido }\end{array}$ & $\begin{array}{c}\text { Correlación total } \\
\text { de elementos } \\
\text { corregida }\end{array}$ & $\begin{array}{c}\text { Alfa de Cronbach } \\
\text { si el elemento se } \\
\text { ha suprimido }\end{array}$ \\
\hline Ítem 1 & 12.48 & 15.925 & .479 & .723 \\
\hline Ítem 2 & 12.57 & 13.299 & .598 & .672 \\
\hline Ítem 3 & 12.71 & 12.965 & .571 & .680 \\
\hline Ítem 4 & 12.84 & 11.529 & .527 & .711 \\
\hline Ítem 5 & 13.21 & 14.639 & .450 & .725 \\
\hline
\end{tabular}

\subsubsection{Funcionamiento: gestión de la participación en el centro}

Para esta parte se obtuvo un valor de .842 . Este resultado indica una elevada consistencia interna y nos permite afirmar que los ítems miden de forma consistente los elementos que se pretenden estudiar. En la tabla 9 se muestra la necesidad de todos los ítems.

Tabla 9. Análisis de cada ítem con el total del subcomponente

"Funcionamiento: gestión de la participación en el centro"

\begin{tabular}{|l|c|c|c|c|}
\hline & $\begin{array}{c}\text { Media de escala si } \\
\text { el elemento se ha } \\
\text { suprimido }\end{array}$ & $\begin{array}{c}\text { Varianza de escala } \\
\text { si el elemento se } \\
\text { ha suprimido }\end{array}$ & $\begin{array}{c}\text { Correlación total } \\
\text { de elementos } \\
\text { corregida }\end{array}$ & $\begin{array}{c}\text { Alfa de Cronbach } \\
\text { si el elemento se } \\
\text { ha suprimido }\end{array}$ \\
\hline Ítem 1 & 12.73 & 11.755 & .476 & .843 \\
\hline Ítem 2 & 12.13 & 10.086 & .714 & .797 \\
\hline Ítem 3 & 12.08 & 10.193 & .694 & .801 \\
\hline Ítem 4 & 11.74 & 11.333 & .582 & .824 \\
\hline Ítem 5 & 11.87 & 10.975 & .627 & .815 \\
\hline Ítem 6 & 11.91 & 10.925 & .631 & .814 \\
\hline
\end{tabular}

\subsubsection{Acceso Universal: aplicación de los principios inclusivos a la participación democrática}

El coeficiente de fiabilidad indicó una elevada consistencia interna, con un valor alfa de .867. Los valores de discriminabilidad para cada ítem presentados en la tabla 10 indican que ninguno de ellos debería ser eliminado en la versión definitiva del cuestionario. 
Tabla 10. Análisis de cada ítem con el total del subcomponente

"Acceso Universal: aplicación de los principios inclusivos a la participación democrática"

\begin{tabular}{|l|c|c|c|c|}
\hline & $\begin{array}{c}\text { Media de escala si } \\
\text { el elemento se ha } \\
\text { suprimido }\end{array}$ & $\begin{array}{c}\text { Varianza de escala } \\
\text { si el elemento se } \\
\text { ha suprimido }\end{array}$ & $\begin{array}{c}\text { Correlación total } \\
\text { de elementos } \\
\text { corregida }\end{array}$ & $\begin{array}{c}\text { Alfa de Cronbach } \\
\text { si el elemento se } \\
\text { ha suprimido }\end{array}$ \\
\hline Ítem 1 & 15.74 & 10.537 & .598 & .856 \\
\hline Ítem 2 & 15.54 & 9.688 & .686 & .840 \\
\hline Ítem 3 & 15.74 & 8.966 & .722 & .833 \\
\hline Ítem 4 & 16.28 & 9.151 & .648 & .840 \\
\hline Ítem 5 & 15.94 & 9.656 & .681 & .841 \\
\hline Ítem 6 & 15.70 & 9.960 & .665 & .844 \\
\hline
\end{tabular}

\subsubsection{Proyección: compromiso del centro en el ámbito de la participación y detección de necesidades}

En este subcomponente se obtuvo un valor de alfa de Cronbach .748. Este resultado nos permite afirmar que los ítems miden de forma consistente los elementos que se pretenden estudiar. Los resultados, que se muestran en la tabla 11, indican que todos los ítems menos el ítem 2 miden de forma consistente los elementos que se pretenden estudiar. El ítem 2 obtendrá mejores resultados con un aumento de la muestra.

Tabla 11. Análisis de cada ítem con el total del subcomponente Proyección: compromiso del centro en el ámbito de la participación y detección de necesidades

\begin{tabular}{|l|c|c|c|c|}
\hline & $\begin{array}{c}\text { Media de escala si } \\
\text { el elemento se ha } \\
\text { suprimido }\end{array}$ & $\begin{array}{c}\text { Varianza de escala } \\
\text { si el elemento se } \\
\text { ha suprimido }\end{array}$ & $\begin{array}{c}\text { Correlación total } \\
\text { de elementos } \\
\text { corregida }\end{array}$ & $\begin{array}{c}\text { Alfa de Cronbach } \\
\text { si el elemento se } \\
\text { ha suprimido }\end{array}$ \\
\hline Ítem 1 & 13.26 & 6.074 & .520 & .703 \\
\hline Ítem 2 & 13.74 & 5.881 & .562 & .690 \\
\hline Ítem 3 & 13.01 & 6.385 & .445 & .722 \\
\hline Ítem 4 & 14.38 & 6.118 & .499 & .708 \\
\hline Ítem 5 & 14.23 & 5.896 & .513 & .704 \\
\hline Ítem 6 & 13.11 & 6.114 & .390 & .742 \\
\hline
\end{tabular}




\section{CONCLUSIONES}

En el presente trabajo hemos presentado la fase previa y piloto de construcción psicométrica de un instrumente que evalúa el rol de los centros educativos con relación a la convivencia y a la participación democrática de la infancia y la adolescencia en vistas a la inclusión e impulso de la voz de los niños y las niñas dentro de la escuela y su proyección al entorno.

Este instrumento ha sido construido en cuatro partes para recoger los elementos que en base a la literatura científica intervienen en la preparación democrática de la infancia y adolescencia (Barnes et al., 2007; Carrillo y Feu, 2015; Charles y Haines, 2014; Cockburn, 2010; Coiduras et al., 2016; Vesterinen et al., 2016; Vis y Fossum, 2015). Las revisiones y valoraciones aportadas por los expertos garantizan que los componentes presentes en el cuestionario representan adecuadamente el contexto del estudio.

Al tratarse de una prueba piloto y una muestra no significativa no es posible presentar los resultados definitivos en cuanto a las propiedades psicométricas. No obstante, apuntamos una primera aproximación con las principales consideraciones que se pueden extraer.

En relación con los dos grandes componentes del cuestionario "Convivencia" y "Participación" se ha calculado la consistencia interna a partir del valor alfa de Cronbach y los resultados indican una alta consistencia interna en ambos. No obstante, considerando los futuros análisis estadísticos inferenciales que se puedan llevar a cabo a partir del instrumento final, se decidió también analizar la consistencia interna de cada subcomponente del cuestionario.

En relación con el componente Convivencia se ha calculado la consistencia interna, a partir del valor alfa de Cronbach, de todos los subcomponentes que lo constituyen (Formación, Prácticas, Funcionamiento, Acceso Universal y Proyección). En todos los subcomponentes los resultados obtenidos son altamente aceptables menos en el subcomponente "Prácticas: programas y acciones para aprender a vivir juntos". Por lo tanto, este subcomponente deberá ser revisado para la versión final del cuestionario. En relación con los ítems 2 y 3 del subcomponente "Funcionamiento: gestión de la convivencia en el centro", se asume que éstos funcionaran de forma adecuada con muestras de mayor tamaño.

En relación con el componente Participación también se ha calculado la consistencia interna, a partir del valor alfa de Cronbach de todos los subcomponentes que lo conforman (Formación, Prácticas, Funcionamiento, Acceso Universal y Proyección). En todos ellos los resultados obtenidos son altamente aceptables y, por lo tanto, se podrán incorporar a la versión definitiva del cuestionario.

Así, para la versión final del instrumento solamente deberá considerarse la revisión del subcomponente "Prácticas: programas y acciones para aprender a vivir juntos" que no se ha ajustado al objetivo pretendido.

\section{REFERENCIAS BIBLIOGRÁFICAS}

Alderson, P. (2010). Younger children's individual participation in "all matters affecting the child." En B. Percy-Smith y N. Thomas, A Handbook of children and young people's participation. Perspectives from theory and practice (pp. 88-96). Routledge.

Álvarez-García, D., Núñez, J. C., Rodríguez, C., Álvarez, L. y Dobarro, A. (2011). Propiedades psicométricas del Cuestionario de Violencia Escolar - Revisado (CUVER). Revista de Psicodidáctica, 16(1), 59-83. 
Barnes, M., Newman, J. y Sullivan, H. (2007). Power, Participation and Political Renewal. The Policy Press.

Belavi, G. y Murillo, F. J. (2016). Educación, democracia y justicia social. Revista Internacional de Educación para la Justicia Social-RIEJS, 5(1), 13-34. doi:10.15336/riejs2016.5.1

Boqué, M. C. (Coord.), Albertí, M., Alguacil, M., Carrillo, E., Gracía Raga, L., Pañellas, M. y Rosich, C. (2014). La construcció de la pau i l'educació per a la ciutadania i els drets humans. Col·lecció Resultats de Recerca, núm. 5. Institut Català Internacional de la Pau. http://icip. gencat.cat/web/.content/continguts/publicacions/documents_i_informes/arxius/la-construcciode-la-pau-i-lepc-i-els-ddhh.pdf

Boqué, M. C., Alguacil, M., García-Raga, L. y Albertí, M. (2017). La contribució dels infants en les societats democràtiques: de la governança de l'escola a la governança del municipi $i$ el país. Investigación financiada por la Generalitat de Cataluña. Departament d'Afers i Relacions Institucionals i Exteriors i Transparència. Ref. 2015DEMOC00016. https://portalrecerca.csuc. cat/cris/project/pj1974328

Bolívar, A. (2016). Educar democráticamente para una ciudadanía activa. Revista Internacional de Educación para la Justicia Social (RIEJS), 5(1), 69-87. http://doi.org/10.15366/riejs2016.5.1

Cameron, C. y Moss, P. (eds.). (2020). Transforming Early Childhood in England: Towards a democratic education. UCL Press https://www.uclpress.co.uk/products/128464

Carbonell, J., Martínez, M., Puig, J. M., Trilla, J. y Uruñuela, P. (2018). Manifiesto por una educación democrática en valores.

http://www.te-feccoo.es/wp-content/uploads/2018/09/MANIFIESTO-por-una-educacióndemocrática-en-valores-8.pdf

Carrillo, I. y Feu, J. (2015). La participació en educació com a expressió de qualitat democràtica. Temps d'educació, 48, 151-172.

Charles, A. y Haines, K. (2014). Measuring Young People's Participation in Decision-Making. The International Journal of Children's Rights, 22(3), 641-659. https://doi.org/10.1163/15718182-55680022

Cockburn, T. (2010). Children and delibertative democracy in England. En B. Percy-Smith y N. Thomas, A Handbook of children and young people's participation. Perspectives from theory and practice (pp. 304-317). Routledge.

Coiduras, J. L., Balsells, M. A., Alsinet, C. y Urrea, A. (2016). La participación del alumnado en la vida del centro: una aproximación desde la comunidad educativa. Revista Complutense de Educación, 27(2), 437-456. https://doi.org/10.5209/rev_RCED.2016.v27.n2.46353

Comisión Europea (2004). Competencias clave para un aprendizaje a lo largo de la vida. Un Marco de referencia europeo. Dirección General de Educación y Cultura (Grupo de trabajo B "Competencias clave").

Consejo de Europa (2010). Carta del Consejo de Europa sobre la educación para la ciudadanía democrática y la educación en Derechos Humanos. Adoptada en el marco de la Recomendación CM/Rec(2010)7 del Comité de Ministros. Departamento de Educación del Consejo de Europa. https://rm.coe.int/1680487829

. (2012). Recomendación del Consejo de Europa sobre la participación de los niños y jóvenes de menos de 18 años de edad. CM/Rec(2012)2. https://rm.coe.int/168046c478

(2015). Declaration on Promoting citizenship and the common values of freedom, tolerance and non-discrimination through education. https://ec.europa.eu/assets/eac/education/ news/2015/documents/citizenship-education-declaration_en.pdf

(2018). Recomendación del Consejo relativa a la promoción de los valores comunes, la educación inclusiva y la dimensión europea de la enseñanza (2018/C 195/01). https://eur-lex. europa.eu/legal-content/ES/TXT/PDF/?uri=CELEX:32018H0607(01)\&from=ES

Cook, S. C. y Rao, K. (2018). Systematically Applying UDL to Effective Practices for Students with Learning Disabilities. Learning Disability Quarterly, 41(3), 179-191. https://doi. org/10.1177/0731948717749936 
Dadich, A. (2010). Participation among young people with mental health issues: redefining the boundaries. En B. Percy-Smith y N. Thomas, A Handbook of children and young people's participation. Perspectives from theory and practice (pp.105-112). Routledge.

Del Rey, R., Casas, J. y Ortega Ruiz, R. (2017). Desarrollo y validación de la Escala de Convivencia Escolar. Universitas Psychologica, 16(1), 1-12. https://doi.org/10.11144/Javeriana.upsy16-1.dvec

Dewey, J. (1916). Democracy and education: An Introduction to the philosophy of education. MacMillan.

Dillon, J., Greenop, D. y Hills, M. (2015). Participation in child protection: A small-scale qualitative study. Qualitative Social Work, 15(1), 1-16. https://doi.org/10.1177/1473325015578946

Echeita, G. y Ainscow, M. (2011). La educación inclusiva como derecho. Marco de referencia y pautas de acción para el desarrollo de una revolución pendiente. Tejuelo. Didáctica de la Lengua y la Literatura. Educación, 12, 26-46. http://hdl.handle.net/10486/661330

Eurydice. (2005). La educación para la ciudadanía en el contexto escolar europeo. Comisión europea.

Feinstein, C., Giertsen, A. y O'Kane, C. (2010). Children's participation in armed conflict and postconflict peace building. En B. Percy-Smith y N. Thomas, A Handbook of children and young people's participation. Perspectives from theory and practice (pp. 53-62). Routledge.

Fielding, M. (2011). La voz del alumnado y la inclusión educativa: una aproximación democrática radical para el aprendizaje intergeneracional. Revista interuniversitaria de formación del profesorado, 70(1), 31-61. https://core.ac.uk/download/pdf/41577483.pdf

Franklin, A. y Sloper, P. (2004). Participation of disabled children and young people in decisionmaking within social services departments in England. Social Policy Research Unit.

Freinet, C. (1996). La escuela moderna francesa. Una pedagogía moderna de sentido común. Las invariantes pedagógicas. Morata.

García-Raga, L. y López Martínez, R. (2014). La convivencia escolar y la construcción de la ciudadanía. Balance retrospectivo y desafíos de futuro, Bordón, 66(2), 93-106. https://doi. org/10.13042/Bordon.2014.66206

Gil-Jaurena, I., Sánchez Melero, H. y López Ronda, S. (2013). La participación como clave en la investigación de espacios de aprendizaje de la ciudadanía. En S. Torío, O. García-Pérez, J. V. Peña y C. M. Fernández (Coords.), Crisis social y el estado del bienestar: las respuestas de la pedagogía social (pp. 84-87). Actas del Congreso Internacional de Pedagogía Social y XXVI Seminario Interuniversitario de Pedagogía Social, Oviedo 7 y 8 de noviembre de 2013.

Gómez Rivas, F. A. (2011). La normativa sobre convivencia escolar en España, Amazónica, 6(1), 149-185.

Griffiths, J., Cunningham G. y Dick, S. (1999). Onwards and upwards: Involving disabled children and young people in decision-making. Children in Scotland.

Kosher, H., Ben-Aryeh, A. y Hendelsman, Y. (2016). Children's Rights and Social Work. Springer.

Liebel, M. (2007). Entre protección y emancipación. Derechos de la infancia y políticas sociales. Facultad de Ciencias Políticas y Sociología.

Liwski, N. (2006). Discurso inaugural del Debate General de Naciones Unidas. http://abc.gov.ar/ lainstitucion/RevistaComponents/Revista/Archivos/anales/numero05/ArchivosParaImprimir/2. liwski.pdf

Martín-Babarro, J., Martínez-Arias, R. y Díaz-Aguado, M. J. (2010). Estudio estatal sobre la convivencia escolar. Ministerio de Educación.

Martín Bris, M. y Gairín, J. (2007). Familias en la educación: un tema por resolver. Bordón, 59(1), 113-151. https://recyt.fecyt.es/index.php/BORDON/article/view/36840

Martínez-Bonafé, M. A. (2016). Els moviments de renovació pedagògica: construint la democràcia de les aules. Educació i Història: Revista d'Història de l'Educació, 27, 83-105.

Moliner, O., Través, J. A., Ruiz, M. P. y Segarra, T. (2016). Estrategias que inciden en los procesos de democratización de la escuela. Una aproximación teórica. Revista Española de Investigación Educativa, 18(2), 116-129. http://redie.uabc.mx/redie/article/view/1110 
Moss, P. (Ed.). (2013). Early Childhood and Compulsory Education: Reconceptualising the relationship. Routledge.

(2014). Transformation Change and Real Utopias: A story of democracy, experimentation and potentiality. Routledge.

Murillo, F. J. y Hernández-Castilla, R. (2014). Liderando escuelas justas para la Justicia Social. Revista Internacional de Educación para la Justicia Social, 3(2), 13-32.

Nunnally, J. C. (1975). Psychometric theory: 25 years ago and now. Educational Researcher, 4, 7-21.

Ochoa, A. y Diez-Martínez, E. (2012). Las interacciones escolares como contexto de construcción de la convivencia en escuelas primarias y secundarias. En Culturas de violencia, culturas de paz. De la reflexión hacia la acción educadores, abogados y defensores de los derechos humanos. Editora CRV.

Ochoa, A. y Salinas de la Vega, J. J. (2016). Entender el conflicto para prevenir la violencia. En J. Carrillo Navarro (Coord.), Las violencias en los entornos escolares (pp. 168-186). Universidad de Guadalajara.

OECD (2020). OECD Future of Education and Skills 2030. Conceptual learning framework. Learning Compass 2030.

https://www.oecd.org/education/2030-project/teaching-and-learning/learning/learningcompass-2030/OECD_Learning_Compass_2030_concept_note.pdf

ONU (1989). Convención sobre los derechos del niño. https://www.un.org/es/events/childrenday/ pdf/derechos.pdf

(2015). Agenda 2030 para el desarrollo sostenible. https://www.un.org/ sustainabledevelopment/es/objetivos-de-desarrollo-sostenible/

Peñalva Vélez, A. y Vega Osés, M. A. (2019). Convivencia escolar, participación y atención a la diversidad: el programa PrInCE. Revista Interuniversitaria de Formación del Profesorado, 33(2), 63-80. https://doi.org/10.47553/rifop.v33i2.72976

Pérez Galván, J. M. y Ochoa, A. (2018). Formación para la ciudadanía y participación infantil. Edetania, 53, 85-98. https://revistas.ucv.es/index.php/Edetania/article/view/344

Rao, K., Bryant, B. y McDougall, D. (2017). Universal design for learning in pre-K to grade 12 classrooms: A systematic review of research. Exceptionality, 25, 116-138. https://doi.org/10.10 80/09362835.2016.1196450

Ray, P. (2010). The participation of children living in the poorest and most difficult situations. En B. Percy-Smith y N. Thomas, A Handbook of children and young people's participation. Perspectives from theory and practice (pp. 63-72). Routledge.

Reddy, N. y Ratna, K. (2002). A Journey in Children's Participation. The Concerned for Working Children.

Sala, I., Sánchez, S., Giné, C. y Díez, E. (2014). Análisis de los distintos enfoques del paradigma del diseño universal aplicado a la educación. Revista Latinoamericana de Educación Inclusiva, 8(1),143-152. http://repositoriocdpd.net:8080/handle/123456789/1971

Santibáñez, D., Delano, J. y Reyes, M. (2015). Participación y ejercicio de derechos. Observatorio niñez y adolescencia.

Santos, M. (2003). Participar es aprender a convivir. En M. Á. Santos Guerra (Coord.), Aprender a convivir en la escuela (pp. 107-122). Akal.

Save the Children (2016). Save the Children's global strategy: ambition for children 2030 and 20162018 strategic plan. Building a better world for and with children.

https://www.savethechildren.net/sites/default/files/Global\%20Strategy\%20-\%20Ambition\%20 for\%20Children\%202030.pdf

Saviani, D. (2018). School and democracy. Autores asociados.

Thomas, N. (Ed.). (2009). Children, politics and communication: Participation at the margins. Policy Press Scholarship Online. https://doi.org/10.1332/policypress/9781847421845.001.0001

Tonucci, F. (1996). La ciudad de los niños: un modo nuevo de pensar la ciudad. Losada-UNICEF Argentina. 
(2003). Cuando los niños dicen ¡basta! Fundación Germán Sánchez Ruipérez.

. (2009). ¿Se puede enseñar la participación? ¿Se puede enseñar la democracia? Investigación en la escuela, 68, 11-24.

Tonucci, F., Renzi, D. y Prisco, A. (2015). El Consejo de los niños. Manual sobre la participación de los niños en el gobierno de la ciudad. Losada.

Toro, B. (2011). Participación y valores ciudadanos. Tesis para la formación política del ciudadano. En B. Toro y A. Tallone (Coords.), Educación Valores y Ciudadanía (pp. 23-28). OIE-Fundación SM.

Trilla, J. y Novella, A. (2011). Participación, democracia y formación para la ciudadanía. Los consejos de infancia. Revista de Educación, 356, 23-43. https://doi.org/10.4438/1988-592X0034-8082-RE

Unión Europea (2014). Horizon 2020. EU Research and Innovation programme. https://ec.europa. eu/programmes/horizon2020/en

Vesterinen, V., Tolppanen, S. y Aksela, M. (2016). Toward citizenship science education: What students do to make the world a better place? International Journal of Science Education, 38(1), 30-50. https://doi.org/10.1080/09500693.2015.1125035

Vis, S. A. y Fossum, S. (2015). Organizational factors and child participation in decision making: differences between two child welfare organizations. Child \& Family Social Work, 20(3), 277 287. https://doi.org/10.1111/cfs.12076

Wanders, F. H. K., van der Veen, I., Dijkstra, A. B. y Maslowski, R. (2020). The influence of teacher-student and student-student relationships on societal involvement in Dutch primary and secondary schools. Theory \& Research in Social Education, 48(1), 101-119. https://doi.org/10. 1080/00933104.2019.1651682 